\title{
Lidil
}

Revue de linguistique et de didactique des langues

$45 \mid 2012$

Pratiques de formation à la lecture-écriture des adultes en parcours d'insertion

\section{Formation aux écrits professionnels : vers une sociolinguistique impliquée}

Hervé Adami et Virginie André

\section{(2) OpenEdition}

Journals

Édition électronique

URL : http://journals.openedition.org/lidil/3178

DOI : $10.4000 /$ lidil. 3178

ISSN : 1960-6052

Éditeur

UGA Éditions/Université Grenoble Alpes

Édition imprimée

Date de publication : 15 mai 2012

Pagination : $51-71$

ISBN : 978-2-84310-226-4

ISSN : $1146-6480$

Référence électronique

Hervé Adami et Virginie André, « Formation aux écrits professionnels : vers une sociolinguistique impliquée », Lidil [En ligne], 45 | 2012, mis en ligne le 15 novembre 2013, consulté le 19 avril 2019. URL : http://journals.openedition.org/lidil/3178; DOI : 10.4000/lidil.3178

(c) Lidil 


\title{
Formation aux écrits professionnels : vers une sociolinguistique impliquée
}

\author{
Hervé Adami et Virginie André*
}

\begin{abstract}
RÉSUMÉ
Cet article présente une recherche en cours qui s'effectue sur le terrain de la formation linguistique à visée professionnelle pour des salariés des métiers de la Propreté. Elle s'intéresse à l'articulation entre la part langagière du travail, la littératie professionnelle, et la formation. Les premiers résultats montrent la difficulté de transformer cette part langagière du travail en contenus didactiques. Au-delà de cette étude, nous montrons qu'il est impossible de comprendre ce qui se passe dans la salle de cours sans remonter tout le fil de l'enquête et des contacts sur le terrain. Cette étude nous amène alors à réfléchir à un nouveau cadre théorique en ce qui concerne l'approche méthodologique que nous proposons d'appeler sociolinguistique impliquée.
\end{abstract}

\section{ABSTRACT}

This article presents ongoing research that is concerned with the provision of language training for employees in the industrial cleaning sector. It deals with the links between the linguistic component of work, literacy in the workplace and the provision of training. Initial results have shown the difficulty arising from trying to deal adequately with the linguistic component of work in terms of language tuition. Beyond this study, we demonstrate here that it is impossible to fully understand what takes place in the classroom without considering the whole of the survey and the contacts in the field. This study invites us to rethink our theoretical framework with regard to the methodological approach; we propose to call this "implied sociolinguistics".

Dans des contextes de travail de plus en plus complexes et dans lesquels la communication ne cesse de croitre, de nombreuses entreprises,

* ATILF, Université de Lorraine, CNRS. Équipe « Acquisition et Apprentissage des Langues ». 
notamment dans les secteurs industriels de la propreté ou du Bâtiment Travaux Publics (BTP), ont pris conscience des problèmes de maitrise de la langue française de certains de leurs salariés les moins qualifiés et/ou migrants. Certaines entreprises ont, par conséquent, mis en place des actions de formation, essentiellement aux écrits professionnels, afin de réduire les problèmes de communication en situation de travail. Cependant, ces nouveaux types de formations au « Français Langue Professionnelle » (Mourlhon-Dallies, 2008), destinés à un public en insécurité langagière, impliquent la mise en œuvre de pratiques spécifiques, s'inscrivant dans une démarche sociolinguistique et sociodidactique. En d'autres termes, l'adéquation des compétences langagières, à acquérir ou à faire acquérir, à la situation de travail et la contextualisation des formations prennent toute leur importance dans ces nouvelles actions formatives. L'étude que nous avons réalisée et que nous présentons ici cherche à mettre au jour l'ensemble des démarches et des actions à accomplir afin de proposer des pratiques de formation appropriées. Pour réaliser cette étude, nous avons notamment observé et analysé des formations aux écrits professionnels mises en place au sein de deux entreprises. Nous verrons que chacun des acteurs de la formation (des décideurs aux apprenants) est amené à repenser son implication et sa façon de concevoir son activité.

Ce travail de recherche de long terme sur le terrain nous a également permis de nous interroger sur des questions méthodologiques et théoriques qui nous semblent consubstantielles et auxquelles nous nous sommes régulièrement heurtés. Nous proposons ici quelques pistes de réflexion à ce propos et un cadre méthodologique utile pour le terrain de recherche que nous investigons mais qui peut, nous le pensons, être exporté sur d'autres terrains.

\section{Des terrains professionnels difficiles d'accès}

Les terrains professionnels qui nous intéressent ici ont fait l'objet de peu de recherches. La formation des adultes en insertion possède une strate de praticiens réflexifs très actifs qui établissent des contacts entre l'université et le terrain mais ils sont d'abord tournés vers l'action et l'ingénierie didactique : leur objectif est de proposer des pistes de réflexion sur les pratiques mais surtout d'apporter une contribution technique aux acteurs de la formation. L'engagement de la recherche institutionnelle sur les terrains de la formation d'adultes en insertion, et notamment les migrants, a toujours été épisodique, pour répondre au coup par coup à 
des demandes sociales ou à l'occasion de débats sociétaux ponctuels (Leclercq, 2010). Le constat du non investissement à long terme de la recherche sur ce terrain a été constaté dans le domaine francophone (Adami, 2009) mais également dans le domaine anglophone (Van de Craats et al., 2006). Même si des recherches commencent à être menées, l'investissement est loin d'être à la hauteur de ce qui est fait dans le domaine du Français Langue Etrangère (FLE) pour les élèves ou les étudiants par exemple. Au-delà de ce constat, quelques explications peuvent être proposées.

Les terrains de la formation d'adultes en insertion sociale et/ou professionnelle sont difficiles d'accès pour des chercheurs pour diverses raisons. Ces terrains sont d'abord caractérisés par l'hétérogénéité institutionnelle des structures qui dispensent les formations : il s'agit d'organismes de formation (OF) à statut associatif ou privé, de services de formations d'entreprises privées ou d'organismes publics et de structures para-publiques comme les GRETA ou l'AFPA par exemple. L'histoire de ces structures et de ce champ éducatif en général, les acteurs et leurs missions, les financements et leurs modalités, les apprenants et leurs objectifs, sont tout à fait particuliers. Or la didactique du français, et toutes ses subdivisions, s'est constituée comme discipline scientifique en partant du terrain scolaire et universitaire dans lequel nous incluons le réseau des Alliances Françaises. Cuq et Gruca (2002 : 14) sont parfaitement clairs à cet égard quand ils proposent de définir le profil prototypique des apprenants de FLE :

Mais qui sont ces apprenants? On peut répondre avec optimisme que tous les étrangers sont des apprenants potentiels de français, mais en réalité certains d'entre eux le sont plus que d'autres :

- des jeunes scolarisés dans des pays qui offrent le français comme choix disciplinaire; des adultes volontaires qui éprouvent un désir ou des besoins linguistiques ou culturels particuliers. C'est à eux qu'on pense en premier lieu et c'est du type de public qu'ils représentent que rend compte l'histoire méthodologique de l'enseignement des langues étrangères dont relève celle du français;

- mais ce sont aussi, et peut-être surtout, ceux qui vivent dans des pays où le français est aujourd'hui présent pour des raisons historiques et politiques.

Le champ de la formation d'adultes en insertion, lui-même hétérogène, est donc structurellement très différent des terrains didactiques de prédilection des chercheurs. 
Ces terrains didactiques, par ailleurs, sont directement connectés à leur environnement économique, social et politique. En effet, tant en ce qui concerne leur organisation, leurs modes de financement que les objectifs d'enseignement liés aux besoins des apprenants et aux exigences des financeurs, les aspects didactiques ne sont pas, et ne sauraient être, définis et considérés per se. Il s'agit donc d'une didactique très fortement contextualisée. Dans ce cadre, l'intervention formative, mais également le travail de recherche, ne peuvent faire abstraction de ce contexte : les praticiens, parce qu'ils ont des objectifs qui en dépendent, notamment en termes d'insertion économique ou sociale, et les chercheurs, qui ne peuvent analyser les situations de formation sans démêler un à un les fils de l'écheveau contextuel. L'analyse des situations de formation nécessite ainsi une forte implication du chercheur sur le terrain qui ne se laisse pas comprendre tout entier par une simple observation, fût-elle fine, de ce qui se passe entre les quatre murs de la salle de classe.

L'évolution récente vers les formations linguistiques à visée professionnelle, pour les migrants ou les personnes en insertion de façon générale, renforce cette nécessité et le recours à l'immersion de longue durée, à la manière des ethnographes, est parfois utilisé (Médina, 2010; Jounin, 2008). Les communautés de pratique (Wenger, 1998) et les communautés épistémiques (Riley, 2002) sont suffisamment structurées dans le milieu professionnel pour en rendre l'accès difficile au chercheur.

Le terrain de la formation linguistique des adultes en insertion, à la fois proche et différent de celui des formations pour enfants et adolescents scolarisés en FLE (Adami, 2005), est ainsi peu investi parce qu'il représente un champ éducatif et social particulier : il n'est pas un terrain didactique comme un autre parce qu'il s'inscrit dans un environnement économique, social et politique auquel il est étroitement connecté. Les recherches en didactique ne peuvent pas simplement s'intéresser aux pratiques de classe mais doivent tenir compte des multiples connections entre ces pratiques et l'environnement dont elles dépendent directement. Ce ne sont pas les seules raisons bien sûr qui font obstacle à la recherche : les publics accueillis, particulièrement hétérogènes, sont eux aussi très mal connus. Bruley-Meszaros (2008 : 42), alors en stage d'observation dans une association, exprime ainsi son désarroi devant la complexité des pratiques à mettre en œuvre : « En effet, même en tant qu'enseignante formée en français langue étrangère (FLE), ce terrain met en évidence le fait que nous ne sommes pas vraiment préparés à ce type de public et c'est pour ça que nous nous interrogeons sur un certain nombre de points. » 


\section{Vers une littératie professionnelle}

La « part langagière du travail » (Boutet, 1995) n'a cessé de croitre depuis déjà plusieurs années, accompagnant de multiples transformations du travail. Ces dernières ont notamment participé à identifier les enjeux de la communication, notamment dans des métiers auparavant considérés comme silencieux. Désormais, rares sont les métiers qui n'engagent pas les salariés dans des pratiques langagières, à l'oral comme à l'écrit. Les discours oraux sont omniprésents en situation de travail. Néanmoins, ils font rarement l'objet de formations spécifiques ${ }^{1}$, l'accent étant mis sur l'écrit tant il apparait élitaire (Goody, 2007). Les formations linguistiques privilégient l'écrit également parce que les rapports à l'écrit des salariés en insécurité langagière sont rares, et que les exigences en termes de compétences ne cessent d'augmenter. Le monde du travail, comme le précise Rouard (2000 : 53) «n'est plus seulement un univers de produits, de services et de processus de fabrication; il est aussi devenu un univers de textes et génère massivement de l'écrit ». Cette littératie professionnelle implique un processus de familiarisation pour des salariés peu habitués à l'écrit et devant acquérir de nouvelles compétences liées à ce que Goody (2007) appelle la «technologie de l'intellect ».

Les activités de littératie professionnelle sont multiples : elles commentent, accompagnent ou constituent les activités de travail (Lacoste, 1995), quel que soit le poste occupé. Les écrits professionnels, qui font l'objet des actions de formation que nous avons analysées dans le cadre de cette étude, prescrivent l'organisation du travail, coordonnent et planifient les tâches à effectuer, diffusent des consignes de travail, d'hygiène et de sécurité, schématisent des ateliers ou des chantiers, décrivent des procédures ou des problèmes. Les écrits professionnels sont également générés par la part réelle du travail ${ }^{2}$, un évènement inattendu ou un problème pouvant être à l'origine d'une activité de lecture ou d'écriture. Les écrits professionnels peuvent donc prendre des formes variées, allant du rapport d'activités d'un travailleur, en quelques pages,

1. Cependant, les contenus de formation, même intitulée « aux écrits professionnels », intègrent généralement un apprentissage des compétences liées aux interactions verbales orales dans la mesure où ces dernières, en situation de travail, requièrent des pratiques spécifiques.

2. Pour une distinction entre travail réel et travail prescrit, voir notamment Leplat et Hoc (1983) ou encore Schwartz (2000). 
au petit papier laissé sur le coin d'un bureau pour signaler un incident. En outre, aujourd'hui, de plus en plus d'entreprises s'engagent dans des démarches de certification et de qualité ou d'obtention de normes ISO (International Organization for Standardization) entrainant une multiplication des procédures de communication et d'information. Ces procédures prennent principalement la forme de documents écrits destinés à l'ensemble des acteurs de l'entreprise, de la direction aux opérateurs. Chaque salarié doit être capable de comprendre et mettre en œuvre les nouvelles exigences de l'organisation impliquée dans ces procédures, et ces exigences sont généralement diffusées par l'intermédiaire d'écrits normés.

La maitrise de la langue est alors envisagée comme une compétence professionnelle incontournable pour répondre aux nouvelles exigences en matière de littératie au travail (Adami et André, 2010). Certains salariés se trouvent dorénavant dans l'obligation d'utiliser l'écrit, par la pratique de la lecture ou de la rédaction, dans leurs activités professionnelles et se voient confier des tâches auparavant réservées au personnel d'encadrement. Par conséquent, les demandes de formation en langue à visée professionnelle (Mourlhon-Dallies, 2008) se multiplient, à l'initiative notamment des OPCA (Organismes Paritaires Collecteurs Agrées) ou des entreprises elles-mêmes. Ces demandes sont impulsées par deux changements, d'ordre idéologique et politique. Le premier est la fin de la perception des problèmes de maitrise de la langue et de l'illettrisme comme un handicap (Fraenkel et Moatty, 2000). Cette perception a freiné la reconnaissance de ces problèmes ainsi que leur prise en charge. Le second est législatif, puisque depuis la loi du 4 mai 2004, relative à la formation professionnelle et à la question des compétences linguistiques et qui intègre le droit à une formation linguistique dans le code du travail, des réflexions portant sur la part langagière des compétences professionnelles ont été engagées et ont fait l'objet de multiples recherches. Les écrits professionnels ont été étudiés par différentes disciplines telles que la linguistique, la sociologie, l'ergonomie ou encore la psychologie du travail notamment au sein du réseau pluridisciplinaire Langage et Travail (Borzeix et Fraenkel, 2001). Néanmoins le lien et l'application de ces études au domaine de la formation restent rares (Lazar, 1998). 


\section{Cadre de l'enquête}

Les premiers travaux de notre groupe de recherche, Langage, Travail et Formation ${ }^{3}$ (LTF), nous ont amenés à nous intéresser à la formation linguistique à visée professionnelle. En effet, la création d'outils d'évaluation des compétences langagières des salariés et des demandeurs d'emploi des métiers de la propreté pour l'OPCA FAF ${ }^{4}$ Propreté (André, 2009) nous a conduits, d'une part, à saisir la part langagière de certaines situations de travail et, d'autre part, à nous interroger sur les formations linguistiques dispensées aux individus échouant aux tests d'évaluation que nous avions créés. Les premières observations de ces formations au Français Langue Professionnelle (FLP) nous ont rapidement interpellés notamment en ce qui concerne l'utilisation, par le formateur, de certains documents ou encore de certaines activités didactiques. À partir de ces observations ponctuelles, nous avons décidé d'entreprendre une étude plus large afin de saisir les enjeux de telles formations pour les demandeurs, les formateurs et les apprenants.

Notre étude nous a amenés à enquêter au sein de deux entreprises de propreté. Conscientes de l'accroissement de la part langagière du travail, ces dernières ont décidé de mettre en place une action de formation aux écrits professionnels pour leurs salariés ayant des difficultés de maitrise de la langue française. La première entreprise, inscrite dans une démarche de certification qualité «MASE » (Manuel d'Amélioration Sécurité des Entreprises), cherchant à réduire les risques au travail, à former et informer le personnel quant à ces risques, intervient dans une industrie lourde. La seconde est une entreprise d'insertion spécialisée dans le nettoyage des bureaux, des administrations et des collectivités. Elle a le statut d'association loi 1901 et propose à ses employés un accompagnement individualisé pour faciliter leur accès à l'emploi durable dans une entreprise traditionnelle. Ces deux entreprises ont fait appel à Formabilis ${ }^{5}$ pour assurer des formations aux écrits professionnels.

Nous avons donc enquêté parallèlement sur les trois terrains impliqués dans la mise en place des formations : les entreprises de propreté,

3. Groupe de recherche de l'axe «Acquisition et apprentissage des langues » de l'ATILF.

4. Fonds d'Actions et de Formations, devenu OPCALIA Propreté depuis le $1^{\text {er }}$ janvier 2012.

5. Formabilis est un organisme de formation et d'insertion. 
les sociétés au sein desquelles ces premières interviennent et l'organisme de formation. Cette investigation nous a permis de saisir les difficultés de la mise en place d'une telle action de formation pour les trois parties prenantes et de dessiner les contours d'une démarche appropriée. L'élaboration de cette dernière, qui semble conduire à la mise en œuvre de pratiques appropriées en formation, nécessite la prise en compte d'un certain nombre de facteurs liés aux spécificités des différentes parties prenantes. En effet, il nous semble important de souligner que ces formations s'insèrent dans des contextes complexes et ont peu de points communs avec des formations linguistiques traditionnelles. Par conséquent, la méthodologie que nous avons mise en place a cherché à recueillir un maximum de données permettant notamment la compréhension des enjeux de la formation.

\section{Quelles ressources théoriques et méthodologiques?}

Les questions de méthodologie de recherche sont dès lors posées : il s'agit à la fois de mener des analyses sur les aspects didactiques proprement dits mais également sur l'environnement économique ou professionnel, social et politique. Si l'on élargit ainsi le champ de recherche, nous intégrons inévitablement des aspects sociolinguistiques, indissociablement mêlés aux aspects didactiques, ce qui conduit par là-même à des investigations longues, à une connaissance du terrain qui nécessite un investissement et une implication de longue durée de type ethnographique. Du point de vue méthodologique, la confrontation avec le terrain de la formation d'adultes en insertion sociale ou professionnelle pour vérifier des hypothèses didactiques générales se heurte bien souvent à l'irréductible spécificité du champ, des acteurs et des apprenants.

Le champ de la formation d'adultes en insertion fait nécessairement appel, pour son analyse, à une contextualisation de sa mise en œuvre, qui va au-delà de la salle de classe. Qu'il s'agisse des pratiques et des conceptions sous-jacentes des formateurs en matière d'intervention didactique, des apprenants et de leurs rapports à l'écrit mais également des contextes institutionnels et économiques dans lesquels ont lieu les formations. L'approche de la littératie par exemple, avec des adultes en insertion, ne se résout ni se réduit à une analyse de type technicienne sur les méthodologies didactiques ou sur les aspects strictement pédagogiques. En formation d'adultes, la dimension « salle de classe » est un artefact qui mutile une réalité complexe et étroitement connectée à son contexte économique, social et politique. 
L'analyse est donc nécessairement multifactorielle et la méthodologie multidimensionnelle. En effet, d'une part la méthodologie ne saurait se limiter à une approche exclusive : les approches qualitatives, quantitatives, de type ethnographique ou de recherche-action n'entrent pas en concurrence mais sont appelées à conjuguer leurs efforts. L'analyse croisée des données recueillies grâce aux différents protocoles d'enquête garantit non seulement une meilleure compréhension des situations mais également une meilleure fiabilité des résultats. D'autre part, une approche de recherche orientée uniquement sur les aspects didactiques ne saurait suffire et là encore, des regards disciplinaires croisés sont nécessaires : didactique des langues et sciences du langage mais également sciences de l'éducation, sociologie, psychologie, ergonomie ou encore didactique professionnelle ${ }^{6}$. Les formations linguistiques des adultes en insertion sociale et/ou professionnelle sont à la croisée de multiples facteurs de nature très différente et aux enjeux éminemment sociaux et pratiques. En conséquence, il nous semble intéressant de réfléchir à une démarche qui s'inscrive dans une approche qui relèverait d'une sociolinguistique impliquée, notion que nous proposons ici à la réflexion.

\section{Une méthodologie multidimensionnelle}

Dans la mesure où notre étude cherche à saisir l'ensemble du cheminement, de la demande institutionnelle à la séance de formation, afin d'envisager une démarche cohérente et appropriée, notre méthodologie d'enquête, de recueil et de traitement des données prend nécessairement de multiples dimensions. Elle s'appuie sur les recommandations de Fraenkel (2001 : 236) pour étudier l'écrit en entreprise et est complétée par d'autres dispositifs. Les différents terrains d'enquête, d'une part, et la complexité des données à analyser, d'autre part, nécessitent une méthodologie multidimensionnelle, c'est-à-dire associant différents types de recueil et d'analyse des données. Ainsi, nous avons mis au point différents protocoles de recueil de données en fonction du terrain et sur un même terrain, nous en avons également élaboré plusieurs.

6. Cette nécessité a d'ailleurs été ressentie par une quinzaine de chercheurs européens d'horizons disciplinaires différents (linguistique, sociolinguistique, didactique des langues, sciences de l'éducation, ergonomie) qui ont décidé de créer un réseau intitulé Langage, Travail et Formation (LTF), du même nom que le groupe de recherche nancéen LTF (ATILF, Université de Lorraine - CNRS) qui en est à l'initiative. 
L'analyse croisée des données recueillies a permis de comprendre les démarches entreprises par les trois partenaires ${ }^{7}$ (entreprises de nettoyage, clients de ces dernières et organisme de formation) et de saisir ainsi les difficultés de ces démarches. Les données n'ont pas été recueillies et analysées de façon successive sur les différents terrains, mais ont été collectées au cours de multiples allers-retours entre ces terrains ${ }^{8}$, d'une part, et entre les différentes analyses et les besoins de la recherche, d'autre part. Nous présentons, ci-dessous, les données recueillies sur les différents sites ainsi que les objectifs des différentes enquêtes.

Tout d'abord, l'investigation des chantiers de nettoyage, au sein des entreprises-clientes, est doublement nécessaire pour, d'une part, appréhender les discours en lien avec l'activité de travail et, d'autre part, tenter d'inscrire les formations linguistiques dans la même perspective contextuelle. Les différentes visites des chantiers et observations des agents de nettoyage pendant leur activité de travail permettent de saisir les communautés de pratiques (Wenger, 1998) s'instaurant notamment avec les différentes façons de communiquer. De façon générale, les recueils de données au sein des entreprises permettent de saisir le rôle de la littératie au travail, ses multiples fonctions (sécurité, hygiène, consignes, répartitions du travail, gestion des problèmes, etc.) dans le contexte précis dans lequel se trouvent les salariés impliqués dans l'action de formation. Nous avons recueilli l'ensemble des documents écrits présents sur les lieux de travail ainsi que les documents auxquels les salariés étaient susceptibles d'accéder ${ }^{9}$. Ce travail d'investigation ethnographique permet de saisir la part langagière du travail des agents de propreté, à l'écrit et à l'oral. Nous avons également suivi les salariés dans leur environnement professionnel pour observer leurs pratiques réelles de lecture et d'écriture, celles que nous ne pouvons pas saisir par la verbalisation de l'activité de travail ${ }^{10}$.

7. Les différentes conceptions de chacun des acteurs de la formation influencent nécessairement cette dernière (voir notamment Escourrou (2011) qui, dans un autre cadre formatif, met au jour l'importance de la pluralité des acteurs de la formation).

8. Nous en profitons pour remercier Aurore Del Mancino, Audrey Musilli et Chloé Radix pour leur investissement sur ces différents terrains d'enquête.

9. Nous avons recueilli 150 pages de documents (feuilles A4), 2 livrets reliés. La quantité de document est inégale selon les sites.

10. Plusieurs vidéos ont été réalisées sur des chantiers pour observer les éventuelles pratiques littératiennes. 
Ensuite, nous avons collaboré avec les entreprises de propreté afin de comprendre la demande de formation aux écrits professionnels et le cadre dans lequel cette formation s'inscrit. La façon dont est appréhendée la formation est importante : elle peut être présentée aux salariés comme une contrainte, une perte de temps pour l'employeur ou, au contraire, comme une valorisation de leur travail. Ce contexte influence nécessairement le déroulement de la formation, notamment en termes d'implication des salariés, d'assiduité, de motivation ou encore simplement d'intérêt et d'utilité ${ }^{11}$.

Enfin, des données textuelles et audio-visuelles ont été recueillies au cours même des formations. Les documents utilisés par les formatrices ont été collectés. Nous avons également observé et filmé les séances de formation, dispensées par trois formatrices différentes : deux formatrices sont intervenues lors de la formation des salariés travaillant dans l'industrie lourde et une seule formatrice a assuré la totalité des interventions pour les salariés travaillant dans le tertiaire. En outre, si ces formations sont particulières et $\mathrm{si}$, nous le verrons, elles requièrent une contextualisation sociale et économique, le public est lui aussi particulier. Nous avons également élaboré un protocole qui nous a permis d'observer les pratiques des stagiaires en formation. Nous avons cherché à observer « ce que les individus font des textes et ce que les activités signifient pour eux » (Barton et Hamilton, 2010 : 48). En d'autres termes, nous avons souhaité analyser les réactions des salariés face aux documents écrits circulant sur leur lieu de travail. Des informations concernant le parcours scolaire, social et professionnel des salariés ont également été recueillies afin de saisir le rapport à l'écrit de ce public particulier. Pendant les séances de formation, les stagiaires ont également été interrogés, lors d'entretiens cherchant à comprendre les besoins communicatifs en situation de travail.

\section{Analyse des données recueillies en formation et en situation professionnelle}

Pour réaliser cette étude, nous avons sélectionné, parmi les données textuelles et audio-visuelles, un peu plus de 12 heures de séances de

11. Nous avons enregistré seulement trois entretiens mais les informations ont notamment été obtenues lors de nombreux échanges informels avec les acteurs des entreprises. 
formation (dispensées par différentes formatrices parmi lesquelles nos étudiantes de master ayant pour objectif d'introduire de nouvelles pratiques), 10 entretiens individuels avec les stagiaires et une centaine de pages de documents professionnels et/ou utilisés en formation. L'analyse de ces données, à la lumière des données situationnelles que nous avons collectées, révèle que les pratiques de formation sont peu adaptées au contexte sociolinguistique et professionnel des salariés, c'est ce que nous montrerons dans un premier temps. Dans un second temps, nous verrons que la mise en place de pratiques appropriées est complexe.

\section{Des pratiques peu contextualisées}

L'analyse des données recueillies, c'est-à-dire l'analyse qualitative des pratiques de formation, l'analyse textuelle des documents recueillis en situation en travail, l'analyse de contenu des entretiens avec les salariés et l'analyse des situations de formation " provoquées $^{12}$ », révèle notamment une inadéquation entre les documents utilisés comme support d'apprentissage et l'environnement professionnel des salariés en formation, c'est-à-dire une inadéquation entre le contenu de la formation et les besoins réels des salariés en termes de littératie au travail. Certaines séances de formation étaient par exemple consacrées à la morphologie du genre des noms (tels que garçon, poupée, livre), à la conjugaison (de verbes tels que manger ou jouer) ou encore à la lecture de texte sur la vie quotidienne. Les documents utilisés par les formatrices sont éloignés des documents susceptibles d'être manipulés par les salariés dans leur situation de travail.

La formation s'inscrit peu dans le contexte sociolinguistique des situations de travail alors que le plan de formation élaboré par l'organisme de formation semble bien approprié aux besoins des salariés. Il semble que les formatrices ne se soient pas inspirées de ce programme. Cet exemple d'ailleurs est une preuve supplémentaire de la complexité du contexte.

\section{Complexité des pratiques sociolinguistiques impliquées}

Le manque de contextualisation des pratiques de formation semble être lié à la complexité des situations (de formation et de travail). Étant donné

12. Nous avions élaboré certaines séances de formation, notamment pour observer les réactions des salariés. 
toutes les démarches, descriptives et analytiques, que nous, chercheurs, avons dû entreprendre pour tenter de définir des pratiques appropriées et cohérentes, nous imaginerons sans peine les difficultés que ce travail peut représenter pour des formateurs astreints à d'autres tâches que strictement pédagogiques (Portefin, 2011 ; Vandermeulen, 2011) et absorbés par la routine parfois non réflexive de l'enseignant au quotidien. Même si, indiscutablement, les formateurs ont des pratiques réflexives (Schön, 1994), ce type de formation, se déroulant sur de courtes périodes, laisse peu de temps aux formateurs pour ajuster leurs pratiques à leur expérience ou pour mener une réflexion sur leur action. En outre, si la démarche est descriptible, chaque situation de formation demande à être contextualisée et les contextes sont toujours différents.

L'investigation longue, notamment pour saisir la réalité globale des chantiers et l'hétérogénéité du public, entreprise par le ou les chercheur(s) devient, dans le cadre de ces formations, quasiment indispensable également pour le formateur ou le concepteur de la formation. La forte contextualisation de ces formations, leur intrication permanente avec l'environnement social, économique et professionnel dans lequel elles s'insèrent, obligent le praticien à devenir ethnographe, sociologue, ergonome, linguiste et didacticien. Sans cette démarche sociolinguistique, prenant en compte les facteurs sociaux influençant les discours en situation de travail ainsi qu'en situation de formation, et sociodidactique, prenant en compte les facteurs sociaux influençant les pratiques d'enseignement et d'apprentissage du FLP, les pratiques des formateurs courent le risque d'être trop fortement décontextualisées. Chacune des variables sociologiques, économiques, linguistiques et didactiques mérite d'être analysée minutieusement et confrontée aux autres pour garantir des actions de formation appropriées.

Le travail des formateurs intervenant dans ces formations linguistiques à visée professionnelle demande des compétences toutes particulières (Mangiante, 2011). Il semble que le formateur doive assumer le rôle d'enquêteur, voire de « para-chercheur ». Il devrait alors pénétrer le monde de l'entreprise, explorer les chantiers et les postes de travail, enregistrer les situations de communication, échanger avec les acteurs de l'entreprise ou encore saisir l'histoire des salariés en découvrant leur biographie. Cependant, il nous parait compliqué, voire impossible, de demander aux formateurs, aussi consciencieux soient-ils, d'accomplir toutes ces démarches et toutes ces analyses. 


\section{L'introduction de documents professionnels : un double défi}

Les premières analyses de cette étude ont été consacrées à la question des documents utilisés en formation. Les observations et les données recueillies ne peuvent être analysées qu'à la lumière de tout ce que nous savons de la situation, de la demande de formation à la mise en place de l'action, en passant par les informations sur chacun des acteurs de cette situation. Le manque d'adéquation entre les formations et les situations de travail effectivement rencontrées par les salariés est notamment dû à l'utilisation, lors des formations, de documents n'ayant pas ou peu de lien avec la réalité professionnelle des stagiaires.

Pour pallier cette décontextualisation des contenus des apprentissages, observée lors de notre enquête, nous avons tout d'abord élaboré des outils, prenant la forme de listes de compétences langagières, pour aider les formateurs à proposer des formations plus appropriées à la situation de travail vécue par les salariés. L'analyse des situations de travail nous a permis de repérer les compétences langagières mises en œuvre et les documents professionnels ont été analysés afin de dégager les compétences nécessaires à leur compréhension et à leur production. L'analyse croisée de l'ensemble des données recueillies dans le cadre de cette étude, comme nous l'avons précisé ci-dessus, a permis de dégager des correspondances entre les compétences langagières à maitriser, celles qui ont été identifiées sur les chantiers notamment, les documents-supports d'apprentissage à exploiter et les activités à créer en fonction des deux premières. Ce type d'outil, recensant les compétences langagières à travailler et la façon de les travailler, est également élaboré en fonction du public visé par la formation, de son niveau de scolarisation et de littératie.

L'introduction de documents professionnels, c'est-à-dire circulant dans l'entreprise et susceptibles d'être manipulés par les salariés, apparait comme une priorité. Néanmoins, nous avons constaté que cette utilisation systématique de documents authentiques met au jour de nouvelles difficultés à la fois pour les formateurs et pour les salariés.

À partir des séances de formation filmées et transcrites, nous avons analysé de quelle façon les salariés abordent les documents écrits professionnels désormais introduits. Nous avons notamment examiné comment ces apprenants migrants et/ou faiblement scolarisés en insécurité linguistique réagissent face à des écrits performatifs (Fraenkel, 2006) présentant une force graphique spécifique (façon d'écrire, choix d'une 
graphie, caractères spéciaux, procédés de mise en forme : gras, italique, souligné, etc.) ainsi que des associations de textes et de tableaux, de graphiques, de photographies, de pictogrammes, etc. Les données concernant les biographies des salariés impliqués dans ces formations prennent tout leur sens ici. Plusieurs dimensions sont à prendre en compte, notamment le parcours scolaire et social des salariés ou encore leurs habitudes en termes de mobilisation cognitive face à une nouvelle ressource textuelle (Barton et Hamilton, 2010). Les salariés doivent désormais être attentifs aux écrits, alors que la majorité d'entre eux l'a rarement été auparavant. Nous observons, lors de la remise aux salariés des documents professionnels recueillis en situation de travail ${ }^{13}$, différentes difficultés liées à un manque de compétences communicationnelles en production écrite. Les salariés présentent des problèmes de compréhension notamment liés à la non maitrise de l'espace graphique (certains documents sont lus dans le mauvais sens par exemple) et à la non reconnaissance de certains éléments permettant d'identifier la fonction du document (les objectifs du document ne sont pas toujours identifiés). D'autres problèmes apparaissent, sémantiques par exemple, lorsque les salariés doivent attribuer un sens à un mot; textuels également, les salariés n'étant parfois pas en mesure de reconnaitre l'organisation d'un texte ou de hiérarchiser des informations.

Parallèlement, nous avons analysé les nouvelles pratiques de formation liées à l'introduction de ces documents authentiques. Tout d'abord, les formatrices ont été réticentes à l'utilisation de ces documents et à la création d'activités à partir de ces derniers. Nous pouvons comprendre ces réactions dans la mesure où nous savons que les formatrices se sont vu refuser l'accès à ces documents, par les entreprises, lors de la préparation de la formation. Nous avons constaté également que les formatrices se sont senties démunies face à des documents inhabituels, complexes et très fortement dépendant de leur contexte d'utilisation alors qu'elles ont chacune été formées pour assurer ces formations. Notre intervention ne représente qu'un aspect de l'étude, nous avons analysé ici la réception de la démarche impliquée que nous proposons. Les pratiques de formation sont effectivement réinterrogées à la lumière de ces nouveaux types d'écrit, éloignés des écrits traditionnellement utilisés en formation

13. Les documents professionnels confiés aux salariés pendant les séances de formation sont accompagnés d'activités avec des objectifs précis d'apprentissage ou de consignes d'évaluation. 
linguistique. C'est l'analyse de tous les facteurs qui influencent les actions de formation qui permet d'adopter une démarche sociolinguistique impliquée. Ainsi, l'analyse des documents permet d'orienter les pratiques de formation mais l'analyse des réactions des apprenants face aux écrits professionnels permet également d'ajuster de nouveau les pratiques de formation ainsi que les pratiques d'apprentissage.

\section{L'implication, entre l'application et l'engagement}

Une sociolinguistique impliquée, au sens que nous lui donnons ici, tente d'éviter deux écueils : d'une part la confusion entre recherche et ingénierie et, d'autre part, entre la recherche et les engagements éthiques.

Sur le premier point, la didactique a dû, pour se constituer comme discipline scientifique, sortir de l'applicationnisme mécaniste qui fait d'elle un simple prolongement de disciplines constituées comme la linguistique surtout. Il est alors question de linguistique appliquée : «Dès 1977, R. Galisson proposa de remplacer linguistique appliquée par didactique des langues étrangères, mais il fallut encore bien des années pour que la didactique se détache réellement de la linguistique, dont elle ne peut être conçue que comme une des applications, sinon des sciences du langage avec lesquelles elle garde encore des liens privilégiés » (Cuq, 2003 : 70). Pour les anglophones, la didactique est encore classée dans les études générales de l'applied linguistics. La didactique ne serait donc rien d'autre qu'une application des théories linguistiques sur un terrain particulier, celui de l'enseignement/apprentissage des langues. La linguistique, dans ce cadre, est la discipline théorique de référence et la didactique une technique, mettant en œuvre ou appliquant ces théories. La didactique est alors réduite à n'être qu'une forme d'ingénierie. De fait, les recherches en didactique, parce que c'est leur vocation même, sont tournées vers le terrain qui est son prolongement obligé.

Pour notre part, nous proposons aujourd'hui une approche sociolinguistique impliquée qui intègre, dans son processus de construction épistémologique, dans ses hypothèses de travail, l'éventualité d'une implication possible sur un terrain d'expérience. Le travail théorique n'est donc plus conçu comme une fin en soi mais comme une référence capable de fournir un cadre en mesure de répondre à d'éventuelles demandes sociales.

Concernant le second point, les publics dont il est question ici, les adultes en insertion sociale et/ou professionnelle, sont davantage les enjeux, souvent à leur corps défendant, de positions, voire de postures 
politiques, que les bénéficiaires d'expertises distanciées, notamment dans le domaine de la formation linguistique des migrants. Le contraste est flagrant en effet entre les innombrables prises de positions généreuses pour la reconnaissance du plurilinguisme, des langues d'origine ou contre les discriminations, réelles ou fantasmées, dont les migrants sont ou seraient les victimes, et l'indigence du nombre de chercheurs qui se sont penchés sur la question concrète et cruciale de la formation. Les débats publics sur la question de l'immigration sont d'une telle intensité depuis une trentaine d'années que la question éthique est inévitablement évoquée même quand le propos général est plutôt technicien (Bretegnier, 2011). Il semblerait que la question de l'engagement colle à la peau de ce champ éducatif qui porte également les marques de son histoire militante (Leclercq, 2010). Dans ce cadre, une sociolinguistique impliquée n'est pas une discipline engagée, au sens militant du terme, parce que ni la science ni les «causes » n'ont à gagner dans cette confusion des genres. Notre approche en revanche a conscience des enjeux sociaux ou économiques de sa recherche, des objets d'étude et des diverses implications des résultats. Elle peut d'ailleurs faire éventuellement des choix politiques, au sens large du terme, en acceptant ou en refusant de mener des travaux dont elle sait que les résultats pourraient être éventuellement utilisés à des fins qui contreviennent aux convictions fondamentales des chercheurs.

Notre approche de la recherche est totalement impliquée dans la vie sociale et prend le risque du contact permanent avec les différents terrains sur lesquels elle est amenée à intervenir. Cependant, la sociolinguistique impliquée n'est au service d'aucune cause parce qu'elle entend maintenir rigoureusement le partage épistémologique entre le domaine de la connaissance et celui de l'opinion. Il ne s'agit pas de mener des recherches qui auraient pour seul objectif de démontrer la validité de telle ou telle opinion, fût-elle tout à fait honorable. Cette position, toujours difficile à assurer et parfois difficile à faire comprendre par les militants de tous horizons, est celle de l'intellectuel spécifique qu' analyse Noiriel (2010).

\section{Conclusion}

L'analyse des pratiques d'enseignement et d'apprentissage dans le cadre des formations aux écrits professionnels ainsi que notre investissement depuis plusieurs années sur le terrain de la formation linguistique à visée professionnelle pour des adultes en insécurité langagière nous ont 
permis d'identifier l'ensemble des éléments contextuels influençant le déroulement de ces formations. Les démarches méthodologiques que nous avons décrites, de l'analyse des demandes de formation à l'appréhension des activités pédagogiques par les salariés, en passant par l'implication et l'action des formateurs, conduisent à mettre en place des pratiques sociolinguistiques et sociodidactiques appropriées.

Une des difficultés majeures dans la mise en place des actions de formations aux écrits professionnels est cette nécessité, encore plus grande que dans des situations de formations « traditionnelles », d'ajustements permanents entre le terrain professionnel, les contenus de formation et le contexte dans lequel cette dernière prend place. Nous avons observé ici que nous pouvons adopter une conception modulaire de la littératie ou des littératies (Street, 2000). Les salariés ont besoin des compétences langagières qui leur permettent de travailler dans les situations qu'ils rencontrent quotidiennement sur leurs chantiers. Les usages des discours écrits nécessitent un traitement particulier en termes d'analyse et de formation afin de garantir l'accès de ces discours aux salariés.

En outre, les analyses que nous avons effectuées lors de cette étude nous amènent à formuler l'hypothèse selon laquelle les compétences acquises dans les formations linguistiques à visée professionnelle semblent également permettre l'intégration et l'insertion de façon plus générale. Bien que les pratiques de l'écrit au travail se différencient des pratiques de l'écrit « vernaculaires », telles qu'elles sont décrites par Barton et Hamilton (2010), elles ont de nombreux aspects en commun. Le travail effectué dans les formations aux écrits professionnels, notamment sur la force graphique, la lecture de tableaux, de schémas, de notes de service, etc. parait réexploitable pour les notes de l'administration, les horaire de bus ou encore les courriers de réclamation. Des transpositions semblent donc possibles, et y compris bien au-delà des littératies professionnelle et quotidienne utilitaires, vers de nouvelles façons de penser, d'agir et d'appréhender la réalité grâce à la maitrise, même partielle, de cette « technologie de l'intellect». 


\section{RÉFÉRENCES BIBLIOGRAPHIQUES}

ADAMI H. (2005) : «Français langue étrangère et enseignement du français aux migrants : les faux-fumeaux didactiques », Le Français dans le monde, $\mathrm{n}^{\circ} 339$, p. 23-26.

ADAMI H. (2009) : La formation linguistique des migrants, Paris, CLE International.

AdAmi H. et ANDRÉ V. (2010) : "Les migrants en insécurité linguistique au travail : analyses et perspectives de formation », Points Communs, $\mathrm{n}^{\circ} 40$, p. $1-5$.

ANDRÉ V. (2009) : «Les compétences langagières des métiers de la propreté : de l'analyse des situations de communication à la formation professionnelle », Bulletin suisse de linguistique appliquée, $\mathrm{n}^{\circ}$ 90, p. 149-165.

Barton D. et Hamilton M. (2010) : « La littératie : une pratique sociale », Langage et société, $\mathrm{n}^{\circ} 133$, p. 45-62.

Borzeix A. et Fraenkel B. (éds) (2001) : Langage et travail : communication, cognition, action, Paris, Les Éditions du CNRS.

Boutet J. (1995) : Paroles au travail, Paris, L'Harmattan.

BRETEGNIER A. (2011) : Formation linguistique en contextes d'insertion. Compétences, posture, professionnalité : concevoir un cadre de référence $(s)$, Berne, Peter Lang.

Bruley-Meszaros C. (2008) : " La réalité des pratiques en milieu associatif. Comment gérer l'hétérogénéité ? Public d'adultes migrants », Les Cahiers de l'Acedle, $\mathrm{n}^{\circ}$ 3, p. 41-66.

CuQ J.-P. (dir.) (2003) : Dictionnaire de didactique du français langue étrangère et seconde, Paris, CLE International.

CuQ J.-P. et Gruca I. (2002) : Cours de didactique du français langue étrangère, Grenoble, Presses universitaires de Grenoble.

Escourrou N. (2011) : «Qui est responsable de la formation : la collectivité, l'entreprise, le formateur ou le stagiaire? »,Éducation permanente, $n^{\circ} 187,2$, p. 95-106.

FraenKel B. (2001) : «Enquêter sur les écrits dans l'organisation », dans A. Borzeix et B. Fraenkel (éds), Langage et travail : communication, cognition, action, Paris, Les Éditions du CNRS, p. 231-261.

FRAENKEL B. (2006) : «Actes écrits, actes oraux : la performativité à l'épreuve de l'écriture », Études de communication, n 29 , p. 69-93.

Fraenkel B. et Moatty F. (2000) : « La mesure de la littératie au travail : résultats, problèmes, perspectives », dans C. El HAYEK (dir.), Illettrisme et monde du travail, Paris, La Documentation française, p. 33-44.

Goody J. (2007) : Pouvoirs et savoirs de l'écrit, Paris, La Dispute.

Jounin N. (2008) : Chantier interdit au public. Enquête parmi les travailleurs $d u$ bâtiment, Paris, La Découverte. 
Lacoste M. (1995) : «Parole, action, situation », dans J. Boutet (dir.), Paroles au travail, Paris, L'Harmattan, p. 23-44.

LAZAR A. (éd.) (1998) : Langage(s) et travail : enjeux de formation. Actes du colloque INRP/CNAM/CNRS-LT, Paris, INRP.

LECLERCQ V. (2010) : « La formation linguistique des migrants des années 1960 aux années $1980 », E ́$ ducation Permanente, n 183, p. 173-188.

LePlat J. et Hoc J.-M. (1983) : « Tâche et activité dans l'analyse psychologique des situations », Cahiers de psychologie cognitive, $\mathrm{n}^{\circ} 3$, p. 49-63.

Mangiante J.-M. (2011) : «Élaborer une analyse de besoins en formation "FLI" : quelles compétences pour les formateurs », dans A. Bretegnier, Formation linguistique en contextes d'insertion. Compétences, posture, professionnalité : concevoir un cadre de référence(s), Berne, Peter Lang, p. 107-120.

MÉdina C. (2010) : «Contextualisation d'un énoncé actionnel en situation professionnelle. Un exemple en Bâtiment et Travaux Publics », dans L. Cadet, J. Goes et J.-M. Mangiante, Langue et intégration. Dimensions institutionnelle, socio-professionnelle et universitaire, p. 275-292.

Mourlhon-Dallies F. (2008) : Enseigner une langue à des fins professionnelles, Paris, Les Éditions Didier.

NoIRIEL G. (2010) : Dire la vérité au pouvoir : les intellectuels en question, Paris, Agone.

PoRTEFIN C. (2011) : «Intervenir auprès d'adultes en "français langue d'insertion" : des formateurs de plus en plus multicartes », dans A. Bretegnier, Formation linguistique en contextes d'insertion. Compétences, posture, professionnalité : concevoir un cadre de référence(s), Berne, Peter Lang, p. 83-92.

RILEY P. (2002) : "Epistemic communities: The social knowledge system, discourse and identity", dans G. Cortese et P. Riley (éds), Domainspecific English: Textual Practices across Communities and Classroom, Berne, Peter Lang, p. 41-64.

RouARD F. (2000) : «Écritures au travail et insécurité linguistique », dans C. El Hayel, Illettrisme et monde du travail, Paris, La Documentation française, p. 53-60.

SchöN D. (1994) : Le praticien réflexif. À la recherche du savoir caché dans l'agir professionnel, Montréal, Éditions logiques.

Schwartz Y. (2000) : Le paradigme ergologique ou un métier de Philosophe, Toulouse, Octares.

Street B. (2000) : "Literacy events and literacy practices. Theory and practice in the New Literacy Studies", dans M. Martin-Jones et K. Jones (éds), Multilingual Literacies, Amsterdam, Johns Benjamins, p. 17-29.

VAn de CraAts I., Kurvers J. et Young-Scholten M. (2006) : Low educated second language and literacy acquisition, Proceedings of the inaugural 
symposium, Tilburg University, août 2005, Utrecht, TOT.

VANDERMEULen K. (2011) : «Formateur dans le champ du français langue d'insertion (FLI) : un métier qui requiert de la polyvalence », dans A. Bretegnier, Formation linguistique en contextes d'insertion. Compétences, posture, professionnalité : concevoir un cadre de référence(s), Berne, Peter Lang, p. 71-81.

WENGER E. (1998) : Communities of Practice. Learning, Meaning and Identity, Cambridge, Cambridge University Press. 
